\title{
Perfil de riesgo de pacientes adultos sometidos a traslado secundario por móviles avanzados del sistema de atención médica de urgencia del Área Metropolitana
}

\author{
MARIE JESIE CARRILLO B. ${ }^{1,2, a}$, MARÍA TERESA URRUTIA S..$^{1, b}$
}

\section{Risk assessment of critical patients requiring ambulance transfers}

Background: Secondary transfers of critical patients between hospitals may be associated with risk of death and complications Aim: To determine the risk profile of adults subjected to secondary transfers using the Emergency Medical Attention System (SAMU) operating in Metropolitan Santiago. Material and Methods: Cross sectional study including 432 adults undergoing secondary transfers using SAMU between January 1 and June 30 2010. Demographic, biomedical, hemodynamic and transfer data were obtained. Cardiopulmonary arrests (CPR) and an increase in the Rapid Emergency Medicine Score (REMS) scale, were considered as outcome variables. Results: CPR occurred in 6.4\% of the study population and was significantly associated with the initial REMS score and the need for hemodynamic and ventilator support. The initial REMS score was a good predictor of the final REMS score. The final REMS was significantly associated with the presence of comorbidities and the need for hemodynamic and ventilator support. Conclusions: REMS is a useful scale to assess the risk profile of critical patients requiring transfers between hospitals.

(Rev Med Chile 2012; 140: 1297-1303).

Key words: Ambulances; Emergency medical services; Risk assessment.

'Escuela de Enfermería,
Pontificia Universidad
Católica de Chile.
2Servicio de Atención
Médica de Urgencia
(SAMU).
aEnfermera Matrona,
Magíster en Enfermería.
bEnfermera Matrona, PhD
Recibido el 16 de diciembre
de 2011, aceptado el 13 de
junio de 2012.
Correspondencia a:
Marie Jesie Carrillo.
Enfermera Matrona,
Instructor, Escuela de
Enfermería,
Pontificia Universidad
Católica de Chile.
Av. Vicuña Mackenna
4860, Macul, Santiago,
Chile
Teléfono: (56-2) 3545837-
3547015
E-mail: mcarrilb@uc.c

'Escuela de Enfermería,

Pontificia Universidad

Católica de Chile.

Médica de Urgencia

(SAMU)

aEnfermera Matrona,

Magíster en Enfermería.

Recibido el 16 de diciembre de 2011, aceptado el 13 de junio de 2012

Correspondencia a: Marie Jesie Carrillo.

Católica de Chile.

Av. Vicuña Mackenna 4860, Macul, Santiago,

Chile

3547015

E-mail:mcarrilb@uc.c
L

a necesidad sanitaria de trasladar pacientes, desde un centro hospitalario a otro, denominada traslado secundario, es una actividad realizada en diversos sistemas de salud. En Chile, el Sistema de Atención Médica de Urgencia (SAMU), como entidad dependiente del Ministerio de Salud, ha enfrentado los requerimientos de la población organizándose para realizar el transporte de pacientes, donde los más críticos son realizados por ambulancias denominadas "avanzadas"1-4.

El traslado secundario, se ha descrito como una actividad que no presenta gran diversidad de causas siendo la mayoría de ellas suplir la falta de recursos humanos o tecnológicos para una adecuada atención del paciente ${ }^{5}$. La decisión de transportar un paciente en estado crítico se basa en una evaluación de los beneficios potenciales del transporte y los posibles riesgos del traslado. Estas decisiones tienen como fin llegar a centros sanitarios que permitan asistencia adicional, ya sea técnica, cognitiva, o de procedimiento, que no se encuentra disponible en el centro de salud de origen ${ }^{6}$. En Estados Unidos de Norteamérica ha sido esencial la regulación del traslado de paciente en estado crítico; desde 1986 a través de las Sociedades Americanas de Cuidados Críticos y de Tratamiento de Emergencia Médica, se han generado normas sobre las responsabilidades de los miembros del equipo de ambulancias, las que incluyen evaluación del paciente previo a la inicia- 
ción del transporte, con el objetivo de garantizar la seguridad de éste durante el traslado ${ }^{7}$. Actualmente, las sociedades americanas, en conjunto con los colegios profesionales de cuidados críticos, establecen la necesidad de determinar los riesgos y los beneficios para el paciente que va a ser sometido a un traslado secundario. La Comisión de Acreditación del Sistema de Transporte Médico, estipula dentro de las normas Norteamericanas la evaluación de la condición hemodinámica del paciente como la primera actividad a realizar previo al traslado ${ }^{8}$. Estas normas son utilizadas en diversos países europeos, que exigen una evaluación estricta de los beneficios potenciales del traslado a un centro de salud versus los potenciales riesgos que adquirirá el paciente, teniendo como objetivo mejorar el pronóstico de éste ${ }^{9-11}$. Utilizar sistemas de clasificación de gravedad, permite identificar grupos de pacientes complejos que requieren un nivel de cuidado mayor durante el traslado ${ }^{12}$.

Los factores que aumentan el riesgo del paciente trasladado se han organizado en base a tres aspectos: Riesgos del transporte, Características biomédicas del paciente, Evaluación del estado de gravedad $^{13}$.

Los riesgos del transporte se basan en cambios bruscos de movimiento (aceleración y desacelaración), detectados por sensores propioceptivos y barorreceptores que desencadenan respuestas correctoras reflejas ${ }^{13}$, movilizar a un paciente conlleva efectos hemodinámicos, que provocan redistribución transitoria de la sangre y otros fluidos, pudiendo ocasionar alteraciones como el aumento de presión venosa central y de la presión intracraneana ${ }^{14}$. Evaluar el riesgo del traslado secundario requiere conocer la duración de éste para determinar alteraciones fisiopatológicas provocadas por los movimientos del transporte terrestre.

Dentro de las características biomédicas, la comorbilidad siempre influye en el pronóstico de gravedad del paciente ${ }^{15}$, proporcionando un sustrato orgánico que favorece la instalación de nuevas enfermedades, lo que genera una condición de desventaja orgánica ${ }^{16}$.

La edad y el género, son condiciones que se relacionan con diversas patologías, un estudio canadiense analizó las diferencias de género y edad en relación a morbimortalidad, concluyendo; en los pacientes mayores de 50 años las mujeres presentan menos probabilidad que los hombres a ser admitidos en la UCI, pero cuando se hospita- lizan, presentan más probabilidad de morir que los hombres ${ }^{17}$.

Otro aspecto a evaluar en el paciente trasladado es el apoyo hemodinámico y ventilatorio requerido durante el transporte, definidos como factores de riesgo durante el traslado según diversas publicaciones ${ }^{18-22}$.

Diversas escalas e índices pronósticos de mortalidad han sido desarrolladas para lograr cuantificar objetivamente la gravedad del paciente crítico y estimar la probabilidad de muerte que presenta según su estado clínico ${ }^{23,24}$. Para simplificar las escalas de gravedad, se han validado "scores" de evaluación rápidos, que no requieren exámenes. Estos "scores" han resultado muy útiles en la evaluación de pacientes que requieren un traslado secundario, dada la necesidad de una valoración rápida del estado de gravedad del paciente ${ }^{25-27}$. El año 2004, se creó el Rapid Emergency Medicine Score (REMS) el que evalúa 6 parámetros ${ }^{28}$, con un rango de puntuación de cada variable de 0-4, excepto para la edad, que es de 0 a 6 , clasificando al paciente en alto riesgo (sobre 13 puntos), intermedio (entre 6 y 13 puntos), bajo riesgo (bajo 6 puntos). Los puntos de cohorte se determinaron por el momento en que se genera aumento de mortalidad, donde el paciente que cae en alto riesgo presenta un riesgo de mortalidad entre un 17 a 20\% ${ }^{29}$. Posteriormente, se validó el REMS para la evaluación de los pacientes en estado crítico que requieren de un traslado, en diversos países ${ }^{11,28,29}$.

El objetivo de esta investigación es la obtención de un perfil de riesgo de los pacientes sometidos a traslado secundario, que permita estratificar grupos de gravedad y disponer de indicadores de calidad de atención con el propósito de mejorar el modelo de gestión en red de los servicios de salud, con énfasis en la estandarización, aumentando la eficiencia, eficacia y efectividad en la resolución de los problemas de salud.

\section{Material y Método}

Se realizó un estudio analítico, observacional de corte transversal en un universo que considera todos los pacientes mayores de 15 años sometidos a trasladados secundarios desde hospitales de alta complejidad tipo III y IV, por ambulancia avanzada de los móviles SAMU del área Metropolitana, entre el $1^{\circ}$ de enero al 30 de junio de 2010. Con 
Perfil de riesgo de pacientes transportados por ambulancia avanzada - M. J. Carrillo et al

95\% de confianza se obtuvo una muestra aleatoria de 432 casos. Se recogieron datos demográficos (edad, género), biomédicos (comorbilidad), hemodinámicos (apoyo hemodinámico y ventilatorio) y del traslado (duración del traslado). Se consideró como variable resultado la presencia de PCR durante el traslado y el aumento de la escala de gravedad REMS. El estudio recibió la aprobación del Comité de Ética de la Escuela de Enfermería de la Pontificia Universidad Católica de Chile. Se realizaron estadísticas descriptivas para las variables categóricas y continuas. Para establecer diferencias entre variables categóricas se aplicó prueba de $\chi^{2}$. Para la comparación de grupos se aplicaron las pruebas $t$ de Student, test Mann-Whitney y ANOVA. Para determinar la correlación entre las variables, la prueba de Pearson. Se elaboraron modelos de regresión logística para determinar las variables asociadas a la presencia de PCR. El análisis de los datos fue por medio del programa estadístico SPSS 18.0. Se consideró significativo un valor $\mathrm{p}<0,05$

\section{Resultados}

La prevalencia de PCR durante el transporte fue de $6,4 \%$. El promedio de edad de los pacientes trasladados fue de $57 \pm 19$ años, siendo $42,8 \%$ de la muestra de sexo femenino. La edad del usuario no mostró diferencias significativas el comparar el grupo de pacientes con y sin PCR, sin embargo, el sexo femenino presentó una prevalencia mayor en el grupo con PCR. El promedio de tiempo de traslado fue en el grupo total de $44 \pm 28 \mathrm{~min}$ (valor $\mathrm{p}=\mathrm{NS}$ ). La existencia de comorbilidad no presentó diferencias significativas entre los grupos, sin embargo, el apoyo hemodinámico (drogas vasoactivas) y apoyo ventilatorio (bolsa manual), fueron significativamente más altos en el grupo de pacientes que presentaron PCR durante el transporte. En relación al REMS inicial (al momento de inicio del traslado) las diferencias fueron significativas entre ambos grupos, señalando un mayor puntaje en aquellos pacientes que desarrollaron un PCR durante el traslado (Tabla 1).

El impacto del traslado se midió mediante el REMS al momento de llegar al hospital de referencia (REMS final). La presencia de comorbilidad y el apoyo hemodinámico y ventilatorio, fueron significativamente más altos en el REMS de riesgo intermedio y alto (Tabla 2). Al analizar el REMS inicial versus el final se pueden observar diferencias significativas entre ambos, mostrando que en la mayoría de los casos el REMS sube su categoría. $\mathrm{Al}$ analizar la correlación entre el REMS inicial y el final se observó que a mayor índice inicial, mayor es el final ( $\mathrm{r}=0,78, \mathrm{p} \leq 0,001)$ (Figura 1).

Finalmente, se realizó un análisis de regresión logística con adición de variables una a una para predecir la ocurrencia de PCR durante el traslado. El modelo propuesto permite estudiar el efecto conjunto de la exposición a distintos factores, controlando el efecto de variables de confusión.

Tabla 1. Características de los pacientes incluidos en el estudio

\begin{tabular}{|lcccc|}
\hline & $\begin{array}{c}\text { Con PCR } \\
(\mathbf{n}=\mathbf{2 7})\end{array}$ & $\begin{array}{c}\text { Sin PCR } \\
(\mathbf{n}=\mathbf{4 0 5})\end{array}$ & $\begin{array}{c}\text { Total } \\
(\mathbf{n}=\mathbf{4 3 2})\end{array}$ & Valor p \\
\hline Edad (años) & $51 \pm 18$ & $57 \pm 19$ & $57 \pm 19$ & NS \\
\hline Género femenino & $61,5 \%$ & $40,4 \%$ & $42,8 \%$ & $0,035^{*}$ \\
\hline Duración traslado (min) & $47 \pm 33$ & $46 \pm 32$ & $44 \pm 28$ & NS \\
Co-morbilidad & $52 \%$ & $58 \%$ & $58,7 \%$ & NS \\
Apoyo hemodinámico & $80,8 \%$ & $27,4 \%$ & $28,9 \%$ & $0,0001^{*}$ \\
Apoyo ventilatorio & $80,8 \%$ & $26,9 \%$ & $29,7 \%$ & $0,0001^{*}$ \\
REMS inicial & & & & $0,0001^{*}$ \\
$\quad-$ Bajo & $11,5 \%$ & $63,6 \%$ & $31 \%$ & $3 \%$ \\
\hline
\end{tabular}

*Valor p significativo. 
Perfil de riesgo de pacientes transportados por ambulancia avanzada - M. J. Carrillo et al

Tabla 2. Factores asociados al aumento del REMS final

\begin{tabular}{|c|c|c|c|c|}
\hline & Bajo (\%) & $\begin{array}{c}\text { REMS final } \\
\text { Intermedio (\%) }\end{array}$ & Alto (\%) & Valor p \\
\hline Femenino & 24 & 55 & 21 & NS \\
\hline Co-morbilidad & $45,1^{\text {a }}$ & $62,5^{b}$ & $60,3^{\mathrm{ab}}$ & $0,009 *$ \\
\hline Apoyo hemodinámico & $19,3^{\mathrm{a}}$ & $27,1^{\text {a }}$ & $59,2^{b}$ & $<0,0001^{*}$ \\
\hline Apoyo ventilatorio & $0,9^{\mathrm{a}}$ & $30^{\mathrm{b}}$ & $76,3^{c}$ & $<0,0001$ * \\
\hline Duración del traslado (min) & $47 \pm 32$ & $43 \pm 24$ & $46 \pm 30$ & NS \\
\hline $\begin{array}{l}\text { REMS inicial } \\
\text { - Bajo } \\
\text { - Intermedio } \\
\text { - Alto }\end{array}$ & $\begin{array}{r}46,5 \\
1,4 \\
--\end{array}$ & $\begin{array}{l}51 \\
50,6 \\
--\end{array}$ & $\begin{array}{l}2,5 \\
39 \\
100\end{array}$ & 0,0001 * \\
\hline
\end{tabular}

* Valor p significativo. a,b,ab,cLos grupos de REM que tienen letras diferentes presentan diferencias estadísticamente significativas $(p<0,05)$, mientras que los que contengan la misma letra no son estadísticamente significativas las diferencias. Así, para Co-morbilidad se tiene que el porcentaje de Co-morbilidad es significativamente inferior en el grupo con REM Bajo que en el grupo con REM Intermedio mientras que el grupo con REM alta está en una posición intermedia que no alcanza a ser significativamente de ninguno de los otros dos grupos. La necesidad de Apoyo Ventilatorio es significativamente diferente entre cualquier par de grupos de REM, es menor en el grupo con REM bajo que en el grupo con REM intermedio, que a su vez es menor que en el grupo con REM alto.

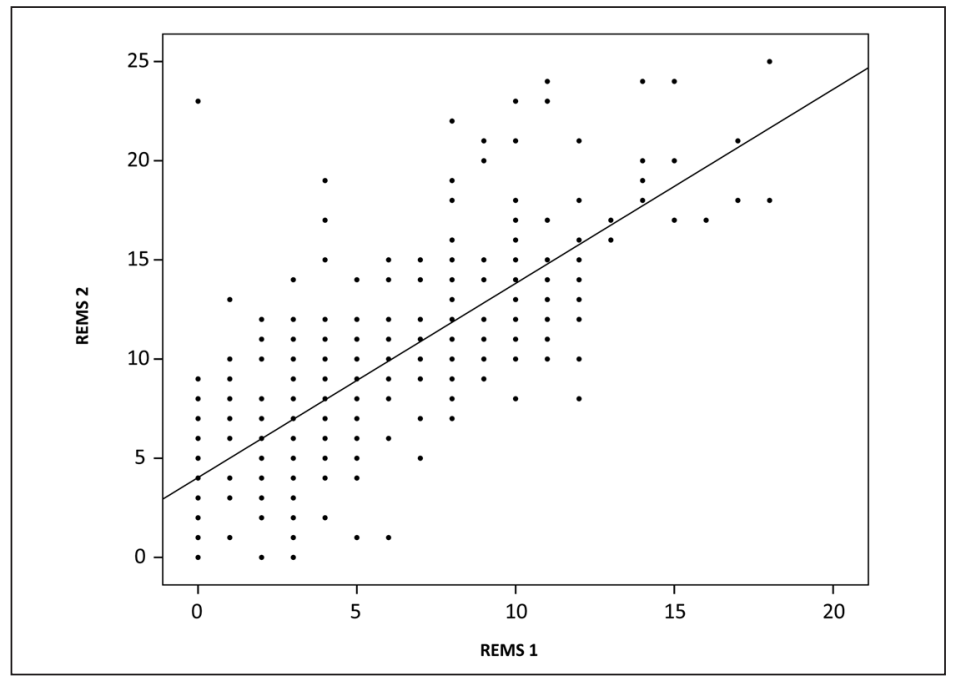

Figura 1. Correlación de Pearson entre REMS inicial y REMS final. REMS 1: REMS inicial. REMS 2: REMS final.
Tabla 3. Modelo de regresión logística para predecir PCR durante el traslado

\begin{tabular}{|lcccc|}
\hline Variables en la & OR & Valor & \multicolumn{2}{c|}{ IC 95\% } \\
ecuación & & p & I. sup. & I. inf. \\
Género (femenino) & 3,7 & $0,014^{*}$ & 1,3 & 10,2 \\
Apoyo hemodinámico & 6,5 & $0,0001^{*}$ & 2,2 & 19 \\
\hline REMS inicial: & & & & \\
Intermedio & 6,6 & $0,004^{*}$ & 1,8 & 24 \\
\hline Alto & 115 & $0,0001^{*}$ & 20 & 679 \\
\hline
\end{tabular}

* Valor $p<0,05$.
Las tres variables que ingresaron al modelo y que por lo tanto, explican el PCR fueron género femenino, REMS (niveles intermedio y alto) y apoyo hemodinámico (Tabla 3 ).

\section{Discusión}

Uno de los aspectos más importantes del presente estudio es que es el primero realizado en Chile que reporta el perfil de riesgo de los pacientes trasladados e identifica las variables 
predictoras del PCR durante el traslado. La principal debilidad es que el estudio considera sólo los eventos adversos ocurridos durante el traslado, no considerando aquellos que se producen durante la hospitalización posterior.

En relación al género de los pacientes trasladados, los resultados señalan la misma tendencia que mostró un estudio canadiense, el que además concluyó que las mujeres cuando se hospitalizan presentan más probabilidad de morir que los hombres ${ }^{17}$. La presente investigación, mostró que en el género femenino la prevalencia de PCR durante el traslado es mayor que en el sexo masculino.

La comorbilidad, influye en el pronóstico de gravedad de un paciente ${ }^{15,16}$. A pesar que el presente estudio no señala asociación entre comorbilidad y PCR durante el transporte, si mostró asociación con el REMS al final del traslado, siendo mayores los porcentajes de comorbilidad en los REMS de tipo intermedio y alto. Por lo anterior se puede señalar que la comorbilidad puede no asociarse a la aparición de un evento adverso inmediato como el PCR, pero si se asocia a un aumento de la gravedad medido por el REMS. Lo descrito muestra la necesidad de realizar estudios que analicen la mortalidad intrahospitalaria de los pacientes que fueron sometidos a traslado secundario, lo que permitirá contar con un perfil de riesgo más completo para poder gestionar mayores recursos según el perfil.

Con respecto al REMS se destaca que 50\% de los grupos de REMS bajo e intermedio subieron de nivel de gravedad, lo que resulta interesante de analizar si se considera que el objetivo principal del traslado secundario es mejorar el pronóstico del paciente ${ }^{11}$. Al respecto, un estudio español comparó el REMS al inicio del traslado secundario $y$ al final de éste, no obteniendo variaciones ${ }^{11}$, sin embargo, en ese estudio, a diferencia de este, 100\% de los traslados se iniciaron con REMS bajos. En la actual investigación, 53,5\% de los pacientes con REMS bajo al inicio del traslado aumentaron su REMS al final de este, lo que muestra una gran variación en el riesgo del paciente, lo que permite concluir que el traslado secundario de la población estudiada aumenta su riesgo de mortalidad en el mayor porcentaje de los casos.

En relación a los factores asociados a un aumento del REMS como son el apoyo hemodinámico y el apoyo ventilatorio, son resultados previamente reportados en estudios de Socieda- des de Cuidados Intensivos internacionales ${ }^{6,19-22}$, quienes señalan a ambas variables como factores de riesgo al momento de trasladar a un paciente.

El 100\% de los traslados de la actual investigación se realizó con ventilación a presión positiva usando bolsa manual, en cambio los traslados de los estudios internacionales fueron con ventilación mecánica, esto podría explicar en algún aspecto el aumento del REMS al final del traslado y la asociación del apoyo ventilatorio con la ocurrencia de PCR dentro de la investigación.

$\mathrm{Al}$ asociar el REMS final con la duración del transporte no se encontraron diferencias significativas. Pareciera que la presencia de carreteras y realizar la investigación en la capital de Chile, región con alto porcentaje de pavimentación de calles y señalética, favorece un traslado con menos complicaciones. Estos resultados concuerdan con investigaciones españolas, mexicanas y Sociedades Médicas de pacientes $\operatorname{críticos}^{11,13,22,25}$.

El presente estudio concluyó que a mayor REMS inicial mayor es la probabilidad de la presencia de PCR durante el transporte, por ende, iniciar el traslado de un paciente con REMS intermedio o alto, requiere de evaluaciones constantes que permitan identificar los recursos necesarios para prevenir la ocurrencia de PCR. Estudios sobre transporte secundario no refieren la ocurrencia de PCR, sólo existen publicaciones de traslados intrahospitalarios que mencionan el PCR como un evento adverso medible. Durante el año 2010, la Sociedad Europea de Medicina Intensiva, además de variados estudios internacionales, identifica al PCR como un evento adverso de los traslados intrahospitalarios de pacientes críticos, con una tasa que va entre $0,34 \%$ a $1,6 \%{ }^{13,30-34}$. El único evento adverso que se pudo medir en nuestro estudio fue la presencia de PCR durante el transporte, el que tuvo una tasa de $6,4 \%$.

Generar un perfil de riesgo, requiere del análisis de los diversos eventos adversos que se generan en relación al paciente, a la organización del transporte y los aspectos técnicos, humanos y colectivos ${ }^{13}$, se debe incluir no sólo las complicaciones inmediatas, sino también las mediatas y tardías de los pacientes trasladados, dentro de las cuales se encuentra la mortalidad intrahospitalaria posterior al transporte.

El aumento del REMS implica un aumento de la mortalidad intrahospitalaria ${ }^{28,29}$, pudiendo resultar una escala útil para medir complicacio- 
nes inmediatas, mediatas y tardías del paciente trasladado. Una de las interrogantes planteadas al comienzo del estudio fue si la gravedad de un paciente podría determinarse por medio del REMS, sin embargo, los parámetros que incluye el REMS estuvieron disponibles en 92,6\% de los casos estudiados, lo que demuestra su factibilidad de aplicación en la realidad nacional. El REMS desarrollado en el contexto pre-hospitalario fue un predictor de PCR en los pacientes, independiente de la edad, diagnóstico de traslado y comorbilidad, por lo que serían necesarias investigaciones nacionales que asocien la gravedad con la mortalidad intrahospitalaria.

Agradecimientos: Las autoras agradecen al Profesor Oslando Padilla P, por su valioso apoyo en el análisis estadístico de los datos.

\section{Referencias}

1. Ministerio de Salud (MINSAL). [Internet] Misión y Visión 2011. [consultado 10 de junio de 2011]. Disponible en: http://www.minsal.gob.cl/portal/url/page/ minsalcl/g_conozcanos/g_mision_vision/presentacion_mision_vision.htm

2. Sanhueza H. División de Gestión Red Asistencial (DIGERA). Encuesta SAMU 2011. [presentación no publicada].

3. García P. Norma técnica de salud número 17. Sobre Sistema de Atención Médica de Urgencia (SAMU): República de Chile Ministerio de Salud. Dpto. Asesoría jurídica; 2005.

4. Ministerio de Salud (MINSAL). Unidad de gestión Centralizada de Camas (UGCC) 2011. [Consultado el 10 de junio de 2011]. Disponible en: http://www.minsal.gob. cl/portal/url/item/a2518ac6a1563fa7e04001011e016979. pdf.

5. Carbajo S, González A, Piedra J, Herrero P, Antuña L, Álvarez B. Transporte sanitario en el traumatismo craneal grave. Emergencias 2002; 14: 143-4.

6. Warren J, Fromm R, Orr R, Rotello L, Horst M. Guidelines for the inter-and intrahospital transport of critically ill patients. Crit Care Med 2004; 32: 256-62.

7. Frew S. Patient transfers. How to comply with the law. JACEP 1990; 12: 8-13.

8. Commission on Accreditation of Medical Transport Systems (CAMTS). [Internet] Accreditation Standards. $8^{\text {th }}$.Edition 2011. [Consultado el 10 de junio de 2011]. Disponible en: http://www.camts.org/component page,shop.product_details/flypage,flypage.tpl/product_
id,5/category_id,2/option,com_virtuemart/Itemid,85/ vmcchk, 11

9. Gray A, Bush S, Whiteley S. Secondary transport of the critically ill and injured adult. Emerg Med J 2004; 21 : 281-5.

10. Vázquez M, Álvarez C. Transporte interhospitalario urgente desde los hospitales comarcales. Revista Emergencias 2008; 20: 245-50.

11. Badia M, Armendáriz J, Vilanova C, Sarmiento O, Servia L Trujillano J. Transporte interhospitalario de largo recorrido. Utilidad de las escalas de gravedad. Med Intensiva 2009; 33: 217-23.

12. Carrillo P, López R, Pinar E, Lozano I, Cortés R, Saura D. Tratamiento del infarto agudo de miocardio con angioplastia primaria in situ frente a transferencia interhospitalaria para su realización: resultados clínicos a corto y largo plazo. Rev Esp Cardiol 2007; 60: 801-10.

13. Fanara B, Manzon C, Barbot O, Demestre T Capellier G. Recommendations for the intra-hospital transport of critically ill patients. Crit Care 2010; 14: 2-10.

14. Márquez E, García S, Chaves J. Transporte de pacientes en estado crítico. En: Barranco F, Blasco J, Mérida A, Muñoz M. Jareño A, Cozar J, Editores, Principios de Urgencia, Emergencia y cuidados críticos. Andaluz, España: edición electrónica; 2002. [Consultado el 10 de junio de 2011]. Disponible en: http://tratado.uninet. edu/indice.htm.

15. Schoenberg MH, Weiss M. Outcome of patients sepsis and septic shock after ICU treatment. Langenbeck's Arch Surg 1998; 283 (1): 44-8.

16. Mayoral M. Sepsis, pronóstico de gravedad y comorbilidad. Rev Fac Med NAM 2003; 46: 193-7.

17. Fowler RA, Sabur N, Li P, Juurlink DN, Pinto R, Hladunewich MA, et al. Sex-and age-based differences in the delivery and outcomes of critical care. CMAJ. 2007; 177: 1513-9.

18. Lahner D, Nikolic A, Marhofer P, Koining H, Germann $\mathrm{P}$, Weinstabl C, et al. Incidence of complications in intrahospital transport of critically ill patients-experience in an Austrian university hospital. Wien Klin Wochenschr 2007; 119: 412-6.

19. Beckmann U, Gillies DM, Berenholtz SM, Wu AW, Pronovost P. Incidents relating to the intra-hospital transfer of critically ill patients. An analysis of the reports submitted to the Australian Incident Monitoring Study in Intensive Care. Intensive Care Med 2004, 30: 1579-85.

20. Damm C, Vandelet P, Petit J, Richard JC, Veber B, Bonmarchand G, Dureuil B. Complications during the intrahospital transport in critically ill patients. Ann Fr Anesth Reanim 2005, 24: 24-30.

21. Markakis C, Dalezios M, Chatzicostas C, Chalkiadaki A, 
Perfil de riesgo de pacientes transportados por ambulancia avanzada - M. J. Carrillo et al

Politi K, Agouridakis PJ. Evaluation of a risk score for interhospital transport of critically ill patients. Emerg Med J 2006; 23: 313-7.

22. Intensive Care Society: Guidelines for the transport of the critically ill adult. 2002. 2002. [Consultado el 10 de junio de 2011]. Disponible en: http://www.ics.ac.uk/ intensive_care_professional/standards_and_guidelines/ transport_of_the_critically_ill_2002.

23. Knaus W A, Zimmerman J E, Wagner D P, Draper E A Lawrence D E. APACHE-acute physiology and chronic health evaluation: a physiologically based classification system. Crit Care Med 1981; 9: 591-7.

24. Knaus W A, Draper E A, Wagner D P \& Zimmerman J E. APACHE II: a severity of disease classification system. Crit Care Med 1985; 13: 818-28.

25. Mondragón E, Díaz E, Rodea H, Pineda P, Sánchez M, Cruz E. Relación de la escala fisiológica aguda con la mortalidad de pacientes adultos trasladados en unidades móviles terrestres. Rev Asoc Mex Med Crit y Ter 2001; 15: 45-50.

26. Rhee K, Mackenzie J, Burney R, Willits N, O`Malley R, Reid N, et al. Rapid acute physiology scoring in transport systems. Crit Care Med 1990; 18: 1119-23.

27. Olsson T, Lind L. Comparison of the Rapid Emergency Medicine Score and APACHE II in nonsurgical emer- gency department patients. Acad Emerg Med 2003; 10: 1040-8.

28. Olsson T, Terent A. Rapid Emergency Medicine Score Can Predict Long-term Mortality in Nonsurgical Emergency Department Patients. Acad Emerg Med 2004; 10: 1008-13.

29. Goodacre S, Turner J, Nicholl J. Prediction of mortality among emergency medical admissions. Emerg Med J 2006; 23: 372-5.

30. Waydhas C. Intrahospital transport of critically ill patients. Crit Care1999, 3: 83-9.

31. Ligtenberg JJ, Arnold LG, Stienstra Y, Van der Werf TS, Meertens JH, Tulleken JE, et al. Quality of interhospital transport of critically ill patients: a prospective audit. Crit Care 2005; 9: 446-51.

32. Taylor JO, Landers CF, Chulay JD, Hood WBJ, Abelmann WH. Monitoringhigh-risk cardiac patients during transportation in hospital. Lancet 1970, II: 1205-8.

33. Papson JP, Russell KL, Taylor DM: Unexpected events during the intrahospital transport of critically ill patients. Acad Emerg Med 2007, 14: 574-7.

34. Damm C, Vandelet P, Petit J, Richard JC, Veber B, Bonmarchand G, Dureuil B. Complications during the intrahospital transport in critically ill patients. Ann Fr Anesth Reanim 2005, 24: 24-30. 